\title{
LA LEGISLACIÓN COOPERATIVA LATINOAMERICANA EN EL MARCO DEL PLAN PARA UNA DÉCADA COOPERATIVA DE LA ALIANZA COOPERATIVA INTERNACIONAL
}

\author{
POR \\ Dante CRACOGNA ${ }^{1}$
}

\section{RESUMEN}

El artículo comienza destacando la importancia de la legislación para el desarrollo de las cooperativas fundándose en el Plan para una Década Cooperativa aprobado por la ACI en su Congreso de Manchester 2012 con el que se clausuró el Año Internacional de las Cooperativas proclamado por las Naciones Unidas bajo el lema "Las cooperativas ayudan a construir un mundo mejor". A partir de allí se ocupa del tratamiento del tema en la propia ACI y en los organismos internacionales para luego referirse a la legislación cooperativa en América Latina analizando sus principales problemas y tendencias actuales dentro del contexto antes desarrollado. Por último, como elementos coadyuvantes al logro de los objetivos del Plan en materia de legislación cooperativa, considera el Proyecto de Ley Marco para las Cooperativas de América Latina y los mecanismos de colaboración establecidos por la ACI Américas y organizaciones cooperativas nacionales con los parlamentarios de la región.

Palabras clave: Cooperativismo, Alianza Cooperativa Internacional, legislación, participación, sostenibilidad, Latinoamérica.

Claves Econlit: P130, K00

\footnotetext{
${ }^{1}$ Profesor consultor de Derecho Comercial de la Universidad de Buenos Aires. Dirección de correo electrónico: dcracogna@estudiocracogna.com.ar.

REVESCO $\mathrm{N}^{\circ} 117$ - MONOGRÁFICO: Las sociedades cooperativas construyen un mundo mejor - ISSN: 1885-8031 - www.ucm.es/info/revesco

http://dx.doi.org/10.5209/rev_REVE.2015.v117.48416

Fecha de recepción: 12/10/2014

Fecha de aceptación: 19/02/2015
} 


\title{
LATIN AMERICAN COOPERATIVE LEGISLATION UNDER THE PLAN FOR A COOPERATIVE DECADE OF INTERNATIONAL ALLIANCE COOPERATIVE
}

\begin{abstract}
This article starts by highlighting the importance of legislation for the development of cooperatives based on the Blueprint for a Cooperative Decade approved by the ICA at its Congress in Manchester 2012, which brought to a close the International Year of Cooperatives proclaimed by the United Nations under the motto "Cooperatives help build a better world". Then, it focuses on the way this issue has been addressed by the ICA itself and international bodies, and refers to cooperative legislation in Latin America by analyzing its main problems and current trends within the above context. Finally, it addresses the Framework Law Project on Cooperatives in Latin America and the collaboration mechanisms established by the ICA Americas and national cooperative organizations with regional members of parliament, as factors that help to achieve the aims of the Blueprint in terms of cooperative legislation.
\end{abstract}

Keywords: Cooperative, cooperative alliance international, law, participation, sustainability, Latin America.

\section{INTRODUCCIÓN}

Cuando se cumplía el centenario de la primera ley de cooperativas del mundo ${ }^{2}$, W.P. Watkins, ex Director de la Alianza Cooperativa Internacional (ACI), escribía: "La auténtica cooperación hunde sus raíces en ámbitos donde no llega el imperio del Estado. Las cooperativas no son creadas por ley. Sin embargo, sin un marco legislativo apropiado no es posible el desarrollo de un movimiento cooperativo sólido." ${ }^{33}$ De manera que la importancia de una adecuada legislación fue reconocida como de significativa relevancia para las cooperativas a lo largo de su existencia, tal como lo señaló L. Valko con relación a la primera cooperativa del mundo en sentido actual de la palabra: "Una vez que el movimiento

\footnotetext{
${ }^{2}$ Industrial and Provident Societies Act de Inglaterra, 1852, cuya sanción fue promovida por los parlamentarios socialcristianos, encabezados por J.M. Ludlow. Merced a ella las cooperativas existentes a partir de la experiencia de Rochdale (1844) pudieron registrarse bajo un régimen propio y diferente del de las Friendly Societies Act (sociedades mutualistas) conforme con el cual hasta ese momento eran legalmente reconocidas (Cfr. Bonner, Arnold, British Co-operation, Co-operative Union, Manchester, 1961, p.66 y ss).

${ }^{3}$ Watkins, W.P., "Preface" to Valko, Laszlo, International Handbook of Cooperative Legislation, State College of Washington, 1954, p. vii. Este libro es uno de los primeros intentos de estudio comparativo de la legislación cooperativa internacional.
}

REVESCO No 117 - MONOGRÁFICO: Las sociedades cooperativas construyen un mundo mejor - ISSN: 1885-8031 - $\underline{\text { www.ucm.es/info/revesco }}$ 
cooperativo estableció una forma exitosa de organización de consumidores debió afrontar permanentes problemas legales. La Cooperativa de Rochdale suministró la organización económica de la empresa cooperativa pero ésta necesitaba imperiosamente una regulación legal apropiada." (Valko, 1952).

De allí que el tema de la legislación cooperativa sea de importancia e interés permanentes puesto que ella constituye un condicionante fundamental para el desarrollo de las cooperativas y del movimiento cooperativo en su conjunto. Las cooperativas nacen y se desenvuelven dentro de una determinada atmósfera legal que les impone exigencias que las condicionan favorable o desfavorablemente y de las que no pueden sustraerse puesto que es característica del derecho su vigencia y aplicación con independencia de la voluntad de los súbditos que habitan en el país donde rige. ${ }^{4}$ Este trabajo intenta un somero análisis de la legislación cooperativa en América Latina como condicionante del cumplimiento de las metas del Plan para una Década Cooperativa formulado por la Alianza Cooperativa Internacional.

\section{EL PLAN PARA UNA DÉCADA COOPERATIVA}

Como colofón del Año Internacional de las Cooperativas (2012) proclamado por la Asamblea General de las Naciones Unidas ${ }^{5}$ bajo el lema "Las cooperativas ayudan a constituir un mundo mejor", la Asamblea Extraordinaria de la ACI realizada en Manchester a fin de octubre de ese mismo año aprobó el Plan para una Década Cooperativa ${ }^{6}$ cuyos objetivos fundamentales consisten en que para el año 2020 el modelo empresarial cooperativo se haya consolidado como:

- El líder reconocido de la sostenibilidad económica, social y medioambiental;

- El modelo preferido por la gente y

- El tipo de organización empresarial de más rápido crecimiento.

Este Plan -que constituye un programa para dar seguimiento al Año Internacional de las Cooperativas- fue producto de una elaboración llevada a cabo por el consejo mundial de la ACI con la colaboración de expertos y sometido finalmente a la asamblea que lo aprobó recomendando algunos ajustes para su redacción final, concluida a comienzos de 2013. A

\footnotetext{
${ }^{4}$ Obviamente, ello no excluye la acción de incidencia que pueda desarrollarse en procura de que los organismos legislativos y regulatorios adecuen las normas a los requerimientos de los diferentes grupos de interés que actúan dentro de la sociedad, incluido el movimiento cooperativo.

${ }^{5}$ ONU, Resolución 64/136, sesión plenaria del 18.12.09.

${ }^{6}$ El Plan para una Década Cooperativa puede ser consultado en www.ica.coop
}

REVESCO No 117 - MONOGRÁFICO: Las sociedades cooperativas construyen un mundo mejor - ISSN: 1885-8031 - www.ucm.es/info/revesco 
partir de entonces el documento alcanzó una amplia difusión con miras a su conocimiento, discusión y aplicación por el movimiento cooperativo mundial.

Comienza el Plan señalando las principales tendencias globales que se advierten en el mundo contemporáneo dentro de cuyo contexto deben desenvolver su actividad las cooperativas. A partir de allí presenta una propuesta de acción futura diseñada para servir de orientación a las cooperativas y sus organizaciones sectoriales, nacionales y regionales con el propósito de alcanzar los objetivos antes señalados.

La estrategia del Plan gira en torno de cinco ejes fundamentales que, convenientemente articulados entre sí, convergen al logro de los objetivos apuntados. Tres de ellos son de carácter conceptual o de contenido, basados en la afirmación de que las cooperativas disponen de una manera de conducir la actividad empresarial mejor que la que ha conducido a la grave crisis en la que se halla sumida la economía a nivel nacional e internacional con sus negativas consecuencias sociales. $^{7}$

En primer lugar, se sostiene que las cooperativas son mejores por cuanto permiten la participación de sus asociados en la propiedad y en la gestión de las empresas, lo cual las ubica en una posición más apta para el progreso económico y social por expresar de manera categórica las aspiraciones de ellos y reconocerles un papel protagónico en la solución de sus necesidades.

Por otra parte, al procurar resolver las necesidades de sus miembros se hallan a cubierto de acciones especulativas que pongan en peligro su estabilidad y permanencia pues fijan su atención en los requerimientos locales (Birchall y Hammond Ketilson, 2009). Todo ello brinda una mayor garantía de continuidad y sostenibilidad ya que deben desenvolver su actividad con respeto de las condiciones económicas, sociales y ambientales del medio en el que actúan pues se trata del ámbito propio de sus integrantes.

Por fin, las cooperativas actúan conforme con valores y principios que definen su naturaleza y su perfil empresarial; es decir que cuentan con una identidad propia que las diferencia de otras clases de empresas y permite transmitir a la sociedad un mensaje distinto que deben enfatizar claramente a fin de evitar confusión.

\footnotetext{
${ }^{7}$ En la introducción del documento se sostiene que "el punto de partida de la estrategia dirigida a un futuro cooperativo se basa en la sólida afirmación expuesta ante el mundo exterior por las cooperativas: que disponen de una manera de conducir la actividad empresarial que es mejor que la actualmente ha fracasado."
}

REVESCO No 117 - MONOGRÁFICO: Las sociedades cooperativas construyen un mundo mejor - ISSN: 1885-8031 - $\underline{\text { www.ucm.es/info/revesco }}$ 
Estos tres ejes: participación, sostenibilidad e identidad, que se refuerzan mutuamente, definen el modelo cooperativo para cuya consolidación y desarrollo se formulan los dos ejes restantes que constituyen factores que facilitan -o limitan, según el caso- la acción de las cooperativas.

El primero de ellos es el marco jurídico que desempeña un papel fundamental en cuanto a la viabilidad y el desenvolvimiento de las cooperativas. El Plan apunta, pues, a establecer lineamientos para la creación de marcos jurídicos que faciliten el desarrollo de las cooperativas.

El segundo de tales factores o ejes de carácter instrumental consiste en la necesidad de que las cooperativas cuenten con capital suficiente para su creación y desenvolvimiento a fin de estar en condiciones de cumplir sus propósitos, lo cual conduce al objetivo de garantizar la existencia de un capital adecuado y confiable cuyo control por parte de los asociados se halle asegurado. $^{8}$

\section{LOS MARCOS JURÍDICOS}

Los cinco ejes incluidos en el Plan son interdependientes y se encuentran relacionados entre sí; pero cada uno de ellos puede ser analizado en forma separada. El objetivo definido en materia de legislación consiste en "Garantizar marcos jurídicos que apoyen el crecimiento cooperativo". El documento comienza señalando que la falta de conocimiento preciso acerca de la naturaleza y funcionamiento de las cooperativas -exacerbada por la ausencia de formación sobre las cooperativas en quienes se incorporan al mundo empresarial- contribuye a que la legislación cooperativa se oriente de manera similar a la de las empresas lucrativas. Ello determina que sean pocos los países que cuentan con una legislación verdaderamente adecuada a las cooperativas por lo cual éstas deben preocuparse por lograrla realizando al efecto una conveniente actividad de incidencia basándose en las directivas de los organismos internacionales. $^{9}$

\footnotetext{
${ }^{8}$ El problema del capital se ha convertido en una preocupación permanente de las cooperativas en la época actual en la que dicho factor devino el motor principal de la actividad empresarial. Ya en la Declaración sobre la Identidad Cooperativa aprobada por el Congreso de Manchester de 1995 la ACI había realizado un reconocimiento explícito del problema cuando incluyó en el texto del $4^{\circ}$ principio que si las cooperativas "reciben capital de fuentes externas, lo hacen en condiciones que garanticen la gestión democrática por parte de sus miembros y respeten su autonomía cooperativa."

${ }^{9}$ La Recomendación 193 de la OIT -a la que se hará referencia más adelante- contiene importantes elementos de orientación al respecto, los que están respaldados por la autoridad del organismo del cual emanó.
}

REVESCO N 117 - MONOGRÁFICO: Las sociedades cooperativas construyen un mundo mejor - ISSN: 1885-8031 - www.ucm.es/info/revesco 
A fin de contribuir al logro de esos objetivos se recomienda colaborar con los reguladores y encargados del registro de cooperativas como así también prestar ayuda a los parlamentarios nacionales y a los responsables de las decisiones de políticas brindándoles la documentación necesaria para apoyar la argumentación en pro de un tratamiento apropiado para las cooperativas -menciona como ejemplo la Ley Marco para las Cooperativas de América Latina- como así también la información conducente al mejor conocimiento de la naturaleza particular de las cooperativas y los beneficios derivados de su actividad. En suma, se promueve una actitud proactiva del movimiento cooperativo y la búsqueda de mecanismos de legislación participativa. ${ }^{10}$

\section{LA LEGISLACIÓN COOPERATIVA EN LA ACI}

Durante muchos años el tema de la legislación cooperativa no ha sido una preocupación central de la ACI. En sus orígenes esta organización se ha preocupado básicamente por construir la unidad entre las cooperativas de diferentes países y actividades. De esa suerte, las cooperativas de consumo inglesas, las cooperativas agrarias y de crédito de Alemania e Italia y las de trabajo de Francia se reconocieron como semejantes entre sí, lo cual permitió afirmar la existencia de un único movimiento cooperativo más allá de las fronteras nacionales y de las actividades específicas de cada sector. La siguiente preocupación consistió en defender, representar y propagar las ideas cooperativas a escala internacional contribuyendo de esa manera a consolidar el movimiento cooperativo. Pero la función principal de la ACI, que constituyó la base de las antes mencionadas, consistió en su labor doctrinaria, es decir en la definición de los principios que caracterizan a las cooperativas y las distinguen de otras organizaciones. Esta acción se orientó inicialmente a establecer los requisitos que debían reunir las entidades que formaban parte de la ACI pero paulatinamente se fue extendiendo con mayor alcance. De manera que lo que constituía la condición para incorporarse a la ACI fue pasando a ser considerada como la caracterización de toda cooperativa. De esa forma la ACI llegó a alcanzar reconocimiento prácticamente indiscutido en el plano internacional como organismo rector en materia de doctrina para todas las cooperativas, con independencia de que estuvieran o no asociadas a ella (Watkins, 1990).

\footnotetext{
${ }^{10}$ La legislación participativa es uno de los objetivos de CLARITY (The Cooperative Law and Regulation Initiative) creada por US Overseas Develompent Council en 2005, la cual lleva realizadas actividades en diferentes países y cuenta con publicaciones sobre el tema que pueden consultarse en www.clarity.coop
}

REVESCO No 117 - MONOGRÁFICO: Las sociedades cooperativas construyen un mundo mejor - ISSN: 1885-8031 - www.ucm.es/info/revesco 
La apuntada circunstancia fue llevando, entonces, a que -aun sin proponérselo la ACI de manera expresa- los principios cooperativos por ella proclamados fueran constituyéndose en una fuente material ${ }^{11}$ de la legislación cooperativa de creciente importancia, influenciado de manera directa o indirecta su contenido. Ello puede constatarse de manera progresiva en la legislación cooperativa a medida que la ACI fue realizando las sucesivas declaraciones de los principios cooperativos en sus Congresos de París 1937, Viena 1966 y, particularmente, Manchester 1995, al punto que en los últimos años se advierten reproducciones casi textuales de aquéllos en distintas leyes de cooperativas. ${ }^{12}$ En efecto, la Declaración sobre la Identidad Cooperativa $^{13}$ aprobada en el mencionado Congreso contiene un plexo integrado por la definición de cooperativa, sus valores y principios que ha servido eficazmente al progreso de la legislación cooperativa en numerosos países. Ello está en línea con la resolución de la asamblea general de la ACI que, al mismo tiempo que aprobó la Declaración, recomendó a las cooperativas incorporarla en sus estatutos y "estimular a sus gobiernos para basar en ella la legislación cooperativa" (ACI, 1995) ${ }^{14}$.

De todas maneras, la ACI también se ha ocupado en forma específica de la legislación cooperativa en diferentes oportunidades, particularmente en la época actual. Así, en varias ocasiones auspició reuniones y estudios sobre la relación entre el Estado y las cooperativas en las cuales la legislación ocupó un lugar destacado ${ }^{15}$, siendo un caso relevante la aprobación en el Congreso realizado en Seúl en 1991 de una recomendación sobre contenidos básicos de la legislación cooperativa (Henrÿ, 2001: pp. 67 y ss.). Asimismo participó activamente en la gestación de los documentos referidos a la legislación cooperativa que fueron aprobados por organismos internacionales, a los que se hará referencia más adelante.

\footnotetext{
${ }^{11}$ En la Teoría General del Derecho se entiende por fuente material a aquellos elementos que son tomados en consideración por el legislador al momento de dictar leyes, no siendo obligatorio que lo hagan como sucede con las fuentes formales (Cueto Rúa, Julio, Fuentes del derecho, Abeledo-Perrot, Buenos Aires, 1994, p. 25).

${ }^{12}$ En las más recientes leyes de América Latina es dable comprobar esta circunstancia, a saber: Venezuela (2001); Uruguay (2008); Ecuador (2011); Bolivia (2013), Honduras (2013). De la misma forma sucede con la Ley Marco para las Cooperativas de América Latina (2008).

${ }^{13}$ Alianza Cooperativa Internacional, Los principios cooperativos para el Siglo XXI, Intercoop, Buenos Aires, 1996.

${ }^{14}$ Resolución de la Asamblea General de la ACI acerca de la Declaración sobre la Identidad Cooperativa, Manchester, septiembre 1995, punto 6, a).

${ }^{15}$ A esas reuniones específicas deben sumarse las Conferencias de Ministros de Cooperativas que en sucesivas oportunidades organizaron las oficinas regionales de la ACI en Asia-Pacífico y África en las que se trataron temas vinculados con la legislación cooperativa en esas regiones. Asimismo en el marco del Congreso del Centenario de la ACI (Manchester, 1995) se llevó a cabo un seminario de la Asociación Internacional de Derecho Cooperativo que fue inaugurado por el Director General de la ACI en el que se trató el tema "Los principios cooperativos en los ordenamientos jurídicos nacionales", tema asimismo del No 23/24 del Boletín de la Asociación Internacional de Derecho Cooperativo, Universidad de Deusto, Bilbao, 1995.
}

REVESCO No 117 - MONOGRÁFICO: Las sociedades cooperativas construyen un mundo mejor - ISSN: 1885-8031 - www.ucm.es/info/revesco 
Más cercana en el tiempo es la resolución de la asamblea extraordinaria de la ACI llevada a cabo en Manchester en 2012 mediante la cual se creó un comité permanente de legislación cooperativa en el seno de la organización. Por fin, la inclusión de los marcos jurídicos dentro del Plan para una Década Cooperativa demuestra de manera elocuente la preocupación actual por el tema puesto que lo incluye dentro del plexo de las cuestiones fundamentales que interesan y condicionan el desarrollo cooperativo de cara al futuro inmediato.

\section{POSICIÓN DE LOS ORGANISMOS INTERNACIONALES}

En 1966 la OIT aprobó la Recomendación N 127 sobre las cooperativas en los países en vías de desarrollo, con especial a las cooperativas agrarias, que contenía importantes orientaciones relacionadas con la legislación cooperativa. Dicha recomendación alcanzó significativa influencia en distintos países.

Sin embargo, ese antecedente fue actualizado y enriquecido a comienzos del presente siglo mediante dos relevantes pronunciamientos que constituyen una base del Derecho Público Internacional Cooperativo como lo señala Hagen Henrÿ. ${ }^{16}$ Se trata de la Resolución 56/114 de la Asamblea General de la ONU de 2001 y la Recomendación N 193 de la OIT sobre la Promoción de las Cooperativas aprobada por la OIT en 2002 en reemplazo de la anterior Recomendación $\mathrm{N}^{\circ} 127$.

La Resolución 56/114 de la $\mathrm{ONU}^{17}$ recomienda a los Estados Miembros unos Lineamientos orientados a la creación de un entorno favorable para el desarrollo de las cooperativas, los cuales se refieren, básicamente, a aspectos de legislación y políticas públicas en materia de cooperativas enfatizando que éstas serán efectivas solamente en la medida que "tomen en consideración el carácter particular de las cooperativas y del movimiento cooperativo, el cual se diferencia notablemente del de las asociaciones y empresas que no están organizadas de acuerdo con los valores y principios cooperativos." Señala la importancia del reconocimiento de las cooperativas en las constituciones nacionales y realiza amplias recomendaciones acerca del contenido de la legislación de la materia, sea ésta de

\footnotetext{
${ }^{16}$ Henry, Hagen afirma que "la Declaración sobre la Identidad Cooperativa de la ACI, los Lineamientos de la ONU y la Recomendación 193 de la OIT, que contiene los valores y principios cooperativos, forman el núcleo del Derecho Público Internacional Cooperativo. Este Derecho debe ser respetado cuando se legisla en materia cooperativa." (Guidelines for Cooperative Legislation, $2^{\text {nd }}$ edition, ILO, Geneva, 2005, p. 5.

${ }^{17}$ UN Document A/56/73-E/2001/68.
}

REVESCO No 117 - MONOGRÁFICO: Las sociedades cooperativas construyen un mundo mejor - ISSN: 1885-8031 - www.ucm.es/info/revesco 
naturaleza general o referida a distintas clases de cooperativas en particular. Asimismo recomienda eliminar las restricciones o discriminaciones legales existentes acerca de las cooperativas y sus actividades. Cabe destacar que los Lineamientos hacen expresa referencia a la Declaración sobre la Identidad Cooperativa adoptada por la ACI en el Congreso de Manchester 1995.

Por su parte, la Recomendación $\mathrm{N}^{\circ} 193$ de la OIT abarca un campo mucho más amplio que su antecesora de 1966 puesto que no se refiere en forma exclusiva a las cooperativas de los países en vías de desarrollo sino a las de todos los países en general y a todas las clases de cooperativas. Si bien contiene disposiciones sobre diferentes aspectos, las referencias a la legislación cooperativa son especialmente significativas. Así, puntualiza que: "los gobiernos deberían establecer una política y un marco jurídico favorables a las cooperativas y compatibles con su naturaleza y función, inspirados en los valores y principios que se enuncian en el párrafo 3" (que son los contenidos en la Declaración sobre la Identidad Cooperativa de la ACI).

De manera que los organismos internacionales no solamente se han preocupado por las cooperativas en términos generales sino que hicieron especial hincapié en la importancia de que los países cuenten con una adecuada legislación cooperativa señalando que para ello deben basarse en la definición, valores y principios proclamados por la ACI. Con ello, queda conformada una verdadera trama coherente de orientación fundada en las definiciones doctrinarias fundamentales adoptadas por la ACI. Por lo tanto, en el mundo actual la legislación cooperativa cuenta con una guía incuestionable para su desarrollo en todos los países.

\section{EL CASO DE AMÉRICA LATINA}

Dentro del contexto señalado cabe ubicar el caso de América Latina a fin de indagar acerca del grado de desarrollo de la legislación cooperativa y su adecuación a los objetivos que en esta materia plantea el Plan para una Década Cooperativa. ${ }^{18}$

Una primera y superficial mirada sobre el estado de la legislación cooperativa en la región permitiría afirmar que ella se ajusta a los lineamientos generales antes mencionados,

\footnotetext{
${ }^{18}$ Sobre el tema en general cfr. Cracogna, Dante, "La promoción del movimiento cooperativo a través de la acción gubernamental en América Latina y el Caribe", Cuadernos de Economía Social, Instituto Argentino de Investigaciones de Economía Social, n 30, Buenos Aires, 1998, p. 85 y ss.
}

REVESCO No 117 - MONOGRÁFICO: Las sociedades cooperativas construyen un mundo mejor - ISSN: 1885-8031 - www.ucm.es/info/revesco 
tal como se hallan expresados en los documentos referidos, tanto emanados del propio movimiento cooperativo como de los organismos internacionales.

En efecto, todos los países cuentan con leyes generales de cooperativas y varios, además, con leyes especiales sobre determinadas clases de cooperativas en particular y no son pocos los que tienen en sus constituciones nacionales cláusulas relativas a las cooperativas.

Por otra parte, todos los países cuentan asimismo con uno o más organismos o agencias gubernamentales encargados de diferentes funciones como la promoción, el registro y, especialmente, la supervisión de las cooperativas. Todo ello podría conducir a considerar que poco es lo que falta hacer en esta materia, máxime cuando diversas leyes acogen de manera expresa los principios proclamados en la Declaración sobre la Identidad Cooperativa.

Sin embargo, una mirada más detenida y profunda sobre la realidad regional permite vislumbrar un panorama que exhibe notables dificultades, a veces por defecto en las legislaciones y otras por anomalías en su aplicación. De algunas de ellas se tratará a continuación, sin que ello implique abarcar a todas ni agotar su consideración. ${ }^{19}$

a. Las constituciones. Si bien es cierto que distintos países incluyen -con variada extensión e intensidad- referencias a las cooperativas en sus constituciones nacionales, existen otros que no las tienen en cuenta. Por lo tanto, de inicio, hay un campo importante para trabajar a fin de lograr que todas las constituciones incorporen estas referencias que resultan de importancia a la hora de legislar sobre las cooperativas puesto que los parlamentos deben hacerlo de conformidad con sus respectivas constituciones. ${ }^{20}$ Además, las disposiciones constitucionales orientan y condicionan la labor de los tribunales; de tal suerte que las normas constitucionales sobre cooperativas deberán asimismo ser tenidas en cuenta en las decisiones jurisprudenciales. ${ }^{21}$

También debe tenerse presente, tanto para los casos de constituciones que contemplan a las cooperativas como de aquéllas que no lo hacen, la diferencia existente entre cláusulas

\footnotetext{
${ }^{19}$ Resultan ilustrativas las reflexiones de José M. Montolío en: Legislación cooperativa mundial. Tendencias y perspectivas en América Latina, Boletín de la Asociación Internacional de Derecho Cooperativo, Bilbao, $\mathrm{N}^{\circ} 45$, 2011, especialmente p. 245 y ss.

${ }^{20}$ El principio de la supremacía constitucional es típico de los sistemas republicanos de gobierno en los cuales la constitución ocupa el pináculo del ordenamiento jurídico como norma supraordinada a todas las demás. (Cfr. Kelsen, Hans, Teoría General del Derecho y el Estado, trad. E. García Maynez, UNAM, México, 1979, p. 246 y ss).

${ }^{21}$ Para garantizar dicha supremacía los ordenamientos nacionales establecen mecanismos de control jurisdiccional sea en forma difusa por cualquier juez o de manera concentrada en un tribunal específico.
}

REVESCO No 117 - MONOGRÁFICO: Las sociedades cooperativas construyen un mundo mejor - ISSN: 1885-8031 - www.ucm.es/info/revesco 
programáticas y operativas. Las primeras consisten en una declaración del constituyente acerca de qué debería ser realizado, sea por el legislador o por el gobierno y, por consiguiente, se hallan supeditadas a que éstos efectivamente dicten las respectivas normas necesarias para su aplicación. Las segundas, en cambio, tienen efecto inmediato; es decir que su vigencia no se encuentra supeditada a que los poderes constituidos decidan aplicarlas. Son numerosas las disposiciones constitucionales sobre cooperativas que se encuadran en el primer caso y, por ende, suelen quedar indefinidamente a la espera de que el legislador resuelva darles operatividad.

b. Caracterización de las cooperativas. Si bien son varias las legislaciones que acogen los principios cooperativos y aun la propia definición de cooperativa de la Declaración sobre la Identidad Cooperativa, hay otras que brindan su propia caracterización de las cooperativas, no siempre en línea con los principios fundamentales que las inspiran. ${ }^{22}$

Resulta evidente que para afirmar la identidad de las cooperativas y distinguirlas claramente de otras organizaciones de diferente naturaleza es necesario que la ley respectiva defina sus rasgos esenciales con precisión, de tal manera que el uso de la denominación "cooperativa" se halle reservado exclusivamente a las entidades que se conforman a esa caracterización. Y que cuando una organización se presente públicamente con el nombre de "cooperativa" sus asociados y los terceros sepan exactamente que ella se corresponde con los principios universales que perfilan a estas organizaciones.

Hace ya varias décadas, a partir de la ley brasileña de 1971, las legislaciones de la región vienen incorporando la noción de "acto cooperativo" para caracterizar a la relación jurídica de particular naturaleza que se establece entre la cooperativa y sus asociados con motivo del cumplimiento del objeto social. Esta disposición contribuye eficazmente a precisar la esencia cooperativa, diferenciándola de la que corresponde a otras entidades de diferente naturaleza y sus efectos ayudan a perfilar mejor los institutos propios del Derecho Cooperativo. $^{23}$

\footnotetext{
${ }^{22}$ Un análisis muy interesante a propósito de este tema, aunque previo a la Declaración sobre la Identidad Cooperativa, puede consultarse en: Münkner, Hans-H., Co-operative Principles and Co-operative Law, Friedrich-Ebert-Stiftung, Bonn, 1985.

${ }^{23}$ Cfr. Cracogna, Dante, "O acto cooperativo na América Latina", en Krueger, Guilherme, Ato Cooperativo e seu adequado tratamento tributário, Mandamentos, Belo Horizonte, 2004, p. 45 y ss.
}

REVESCO No 117 - MONOGRÁFICO: Las sociedades cooperativas construyen un mundo mejor - ISSN: 1885-8031 - www.ucm.es/info/revesco 
c. El registro o constitución legal de las cooperativas. Aspecto de singular importancia constituye el reconocimiento legal de las cooperativas como sujetos de derecho o personas jurídicas a fin de que puedan actuar con status legal diferente de sus integrantes. Todavía son mayoría los países que aplican el régimen de la "autorización", es decir que sujetan ese reconocimiento a una decisión voluntaria de un organismo del gobierno que puede exigir el cumplimiento de excesivos requisitos a tal efecto o bien demorar la resolución durante un tiempo tan prolongado que desalienta a los interesados.

Son relativamente pocos los países que aplican el sistema del "registro" consistente en que el organismo encargado de esa función se limite a comprobar que la cooperativa que solicita su reconocimiento haya cumplido con los requisitos legalmente establecidos para su inscripción, con lo cual queda reconocida como persona jurídica.

En cambio, el reconocimiento, incorporación o constitución legal de las sociedades comerciales suele realizarse en forma mucho más rápida y expeditiva, sujeto a menores recaudos, con lo cual indirectamente se induce a la adopción de esas formas jurídicas que resultan más sencillas y ágiles. Curiosamente, se suele afirmar que las mayores exigencias que se plantean a las cooperativas se fundan en la necesidad de tutela estatal, la cual deviene contraproducente. ${ }^{24}$ La inexistencia o dificultad para acceder al control judicial de las decisiones de los organismos administrativos competentes agrava aún más este problema.

d. Relación con el Estado. Cuestión de relevante significación ha sido, históricamente, la concerniente a la relación entre el Estado y las cooperativas, aspecto que no preocupa a las sociedades comerciales. Sucede que por ser las cooperativas entidades que, además de perseguir la satisfacción de necesidades de sus asociados, tienen preocupación por la comunidad ${ }^{25}$, han solido ser consideradas como instrumentos de desarrollo que podían ser utilizadas por el Estado para el cumplimiento de sus finalidades de interés general (Cracogna, 1993).

Esta actitud del Estado asumió diferentes grados: desde la absorción de las cooperativas dentro del aparato de la economía centralmente planificada, al estilo soviético,

\footnotetext{
${ }^{24}$ La Recomendación $N^{\circ} 193$ de la OIT expresamente indica "establecer un marco institucional que permita proceder al registro de las cooperativas de la manera más rápida, sencilla, económica y eficaz posible" (punto 6. a).

${ }^{25}$ El $7{ }^{\circ}$ principio consagrado por la Declaración sobre la Identidad Cooperativa reza: "A la vez que atienden las necesidades de sus asociados, las cooperativas trabajan en pro del desarrollo sostenible de sus comunidades mediante políticas aprobadas por aquéllos."
}

REVESCO No 117 - MONOGRÁFICO: Las sociedades cooperativas construyen un mundo mejor - ISSN: 1885-8031 - www.ucm.es/info/revesco 
hasta la consideración de las cooperativas como un medio de promoción del desarrollo de regiones y sectores a través del apoyo estatal, como sucedió en la India mediante la Ley de Cooperativas de Crédito de 1904 y la posterior Ley General de Cooperativas de 1912.

Los resultados fueron, obviamente, diferentes, toda vez que las cooperativas del régimen soviético perdieron su genuina condición de tales convirtiéndose en un mero apéndice de la estructura del Estado, en tanto que las cooperativas de la India y de los países que siguieron ese modelo se desarrollaron en forma autónoma y trabajaron en asociación con el Estado sin perder su naturaleza. ${ }^{26}$

En América Latina esta cuestión no se halla resuelta en unos cuantos países en los que el Estado insiste en considerar a las cooperativas como ejecutoras de sus políticas en materia económica y social. De allí que promueva su creación con esa finalidad asignándoles, incluso, recursos públicos, pero sujetándolas a sus directivas y políticas. Esta situación conflictúa abiertamente con los principios de ingreso libre y voluntario de los asociados, de control democrático y, especialmente, de autonomía e independencia. Suele darse la paradoja de que la ley reconoce la autonomía de las cooperativas, pero las políticas del gobierno las instrumentan como ejecutoras de sus programas.

e. La supervisión estatal: Estrechamente vinculado con el punto anterior se encuentra el tema de la supervisión o fiscalización estatal de las cooperativas. Ocurre con marcada frecuencia que las legislaciones y regulaciones administrativas imponen a las cooperativas medidas de fiscalización que son mucho más gravosas que las que corresponden a las sociedades comerciales, llegando a convertirse en mecanismos que las colocan en seria desventaja frente a aquéllas. Otra vez, el argumento con que suele pretenderse justificar esta situación es -curiosamente- la tutela y protección del funcionamiento de las cooperativas. ${ }^{27}$

La Recomendación $N^{\circ} 193$ de la OIT es categórica en esta materia en cuanto insta a los gobiernos a "prever la adopción de medidas de supervisión de las cooperativas acordes con su naturaleza y funciones, que respeten su autonomía y sean conformes con la legislación y la práctica nacionales y no menos favorables que las medidas aplicables a otras formas de

\footnotetext{
${ }^{26}$ Un amplio y didáctico desarrollo del tema se encuentra en: Münkner, Hans-H., "The 'Classical British Indian Pattern of Co-operation' from state-sponsorship to state-control", en Münkner, Hans-H. (Editor), 100 Years Cooperative Credit Societies Act India 1904. A worldwide applied model of co-operative legislation, Marburg Consult for Self-help Promotion, Marburg, 2005, p. 73 y ss.

${ }^{27}$ Cfr. Cracogna, Dante, Estado, cooperativas y legislación cooperativa en la hora actual, Boletín de la Asociación Internacional de Derecho Cooperativo, $\mathrm{N}^{\circ}$ 47, Bilbao, 2013, p. 111 y ss.
}

REVESCO No 117 - MONOGRÁFICO: Las sociedades cooperativas construyen un mundo mejor - ISSN: 1885-8031 - www.ucm.es/info/revesco 
empresa y de organización social." ${ }^{28}$ De manera semejante se expiden los Lineamientos contenidos en la Resolución 56/114 de la ONU. ${ }^{29}$

Por último, cabe señalar que la supervisión por razón de la actividad de las cooperativas que realizan organismos especializados como las superintendencias de banca y de seguros y otros similares comúnmente no toman en consideración las características propias de las cooperativas como organizaciones constituidas por usuarios de esos servicios y llegan a plantear exigencias contrarias a su naturaleza.

f. La integración cooperativa. Son numerosos los países cuya legislación regula la posibilidad de que las cooperativas constituyan organizaciones de grado superior: federaciones, centrales, uniones, confederaciones, etc., distinguiendo, por lo común, la integración con fines económicos y la integración con propósitos de representación.

A pesar de que la integración o colaboración entre cooperativas es un principio cooperativo que, en cuanto tal, debería estar reconocido por la ley, sucede a veces que existen trabas o restricciones legales para su implementación o bien que ella se encuentra impuesta de manera obligatoria, especialmente en materia de representación. De esta suerte, en lugar de ser una forma libre y voluntaria de relación entre cooperativas para servir mejor a sus asociados, se convierte en un mecanismo forzoso que la ley exige y que suele estar subordinado al organismo o agencia gubernamental de cooperativas.

g. Limitación de actividades. Una frecuente restricción que las leyes suelen imponer al desarrollo de las cooperativas consiste en reservar determinadas actividades en forma exclusiva a las sociedades comerciales, particularmente a las sociedades por acciones. Esta limitación suele tener lugar en materia de bancos, seguros, comercio exterior y medios de comunicación, principalmente.

Esta discriminación negativa no encuentra asidero racional puesto que en muchos países del mundo las cooperativas realizan tales actividades en pie de igualdad con las empresas comerciales demostrando con toda evidencia su capacidad técnica y económica de llevarlas adelante eficientemente en beneficio de sus asociados. Por ello no se entiende el motivo de la prohibición.

\footnotetext{
${ }^{28}$ OIT, Recomendación $N^{\circ} 193$, punto 6. c).

${ }^{29}$ ONU, Lineamientos, punto 11 .

REVESCO No 117 - MONOGRÁFICO: Las sociedades cooperativas construyen un mundo mejor - ISSN: 1885-8031 - www.ucm.es/info/revesco
} 
Los Lineamientos de la ONU expresamente establecen que los gobiernos deben excluir o eliminar las disposiciones de cualquier ley que discrimine en contra o sea específicamente perjudicial para las cooperativas. ${ }^{30}$ El texto es claro en cuanto se refiere no solamente a la ley de cooperativas sino a cualquier otra pues suele suceder que las restricciones se hallan contenidas en diferentes disposiciones legales. Por ello recomienda también que los gobiernos creen condiciones adecuadas para posibilitar que las cooperativas puedan denunciar estas situaciones.

h. Capital y financiamiento. El acceso a mecanismos adecuados de financiamiento es una necesidad que se plantea a las cooperativas como a cualquier otra forma de empresa. Sin embargo, es corriente que se impongan a las cooperativas limitaciones que no afectan a las empresas comerciales lucrativas. La posibilidad de asumir formas de endeudamiento comunes en el mercado de capitales debe estar abierta a las cooperativas en pie de igualdad con el resto de las organizaciones empresarias.

Por otra parte, el capital propio de la cooperativa ha de contar con un tratamiento conforme con su naturaleza de aporte de riesgo que realizan los asociados para dotarla de un patrimonio apto para posibilitar su desenvolvimiento económico financiero. De allí que el tratamiento contable del capital cooperativo deba ser el de un genuino recurso patrimonial que los asociados efectúan y no de un mero pasivo. ${ }^{31}$

Por otro lado, en algunas legislaciones se prevé la existencia de capital aportado por asociados no usuarios de los servicios de la cooperativa, tema que debe ser analizado cuidadosamente para evitar la distorsión de la figura cooperativa, sobre todo cuando se les reconoce a tales asociados derecho de voto en las asambleas y participación en los órganos de administración y fiscalización. Como ya se recordó, el $4^{\circ}$ principio contenido en la Declaración sobre la Identidad Cooperativa expresamente establece que si las cooperativas "captan capital de fuentes externas, lo hacen en términos que aseguran el control por parte de los asociados y mantienen su autonomía cooperativa."

Existen variadas maneras de facilitar el incremento del capital propio mediante la formación de fondos rotatorios, capital proporcional al uso de los servicios, aportes

\footnotetext{
${ }^{30}$ ONU, Lineamientos, punto 14.

${ }^{31}$ Cfr. Cracogna, Dante, El capital en las cooperativas y las NIIF. En Favier Dubois, Eduardo M. (Director), Derecho contable aplicado, Errepar, Buenos Aires, 2012, p. 369 y ss.
}

REVESCO No 117 - MONOGRÁFICO: Las sociedades cooperativas construyen un mundo mejor - ISSN: 1885-8031 - www.ucm.es/info/revesco 
extraordinarios, reconocimiento de un interés atractivo, etc. y, por cierto, la constitución de reservas acumulables que paulatinamente suplementan el capital y reducen el costo financiero. La incorporación como asociados de entidades no lucrativas, agencias de desarrollo, otras cooperativas, y hasta del propio Estado bajo determinadas condiciones, puede asimismo constituir una interesante fuente de financiamiento que no comprometa la autonomía de la cooperativa. $^{32}$

Finalmente, cabe recordar que el $5^{\circ}$ objetivo del Plan para una Década Cooperativa consiste en: "Obtener capital fiable para las cooperativas al mismo tiempo que se garantice el control por parte de los asociados."

i. Las cooperativas de trabajo. La crisis económica y los cambios tecnológicos han provocado un grave problema de desempleo que azota por igual a los países industrializados como a las naciones en desarrollo. Dentro de ese contexto las cooperativas de trabajo asociado han cobrado una relevancia particular. En efecto, mientras que tradicionalmente estas cooperativas constituían un sector menor dentro del movimiento cooperativo, circunscripto a solamente algunos casos de organizaciones de trabajadores que espontáneamente recurrían a esta organización como forma superadora de la empresa convencional fundada en la relación obrero-patrón, ahora las cosas exhiben un cariz diferente.

Las situaciones que se presentan suelen ser las siguientes: a) por una parte, las cooperativas de trabajo surgen como respuesta al desempleo procurando un cauce organizativo a grupos de emprendedores carentes de inserción laboral o que procuran iniciar una nueva actividad por su propia cuenta; b) otro caso es el de empresas convencionales que caen en estado de insolvencia o quiebra, frente al cual los trabajadores resuelven hacerse cargo de la empresa como medio de conservar su fuente de trabajo ${ }^{33}$ y c) las cooperativas de trabajo organizadas por el Estado como parte de las políticas sociales orientadas a paliar el

\footnotetext{
${ }^{32}$ La problemática del capital y el financiamiento cooperativo ha motivado una importante producción bibliográfica en los últimos años. Resultan ilustrativos al respecto: Celaya Ulibarri, Adrián, Capital y sociedad cooperativa, Tecnos, Madrid, 1992 y Pastor Sempere, Carmen, Los recursos propios en las sociedades cooperativas, Editoriales de Derecho Reunidas, Madrid, 2002.

${ }^{33}$ Es el caso de las llamadas "empresas recuperadas", acerca de las cuales existen experiencias en diferentes países. Para el caso de Argentina, donde el fenómeno ha sido particularmente importante a partir de la crisis de 2001, puede consultarse: Tevez, Alejandra Noemí, Empresas recuperadas y cooperativas de trabajo, Astrea, Buenos Aires, 2010 y Fontenla, Eduardo H., Cooperativas de trabajo y empresas recuperadas, Intercoop, Buenos Aires, 2008.
}

REVESCO N 117 - MONOGRÁFICO: Las sociedades cooperativas construyen un mundo mejor - ISSN: 1885-8031 - www.ucm.es/info/revesco 
desempleo. ${ }^{34}$ En los dos últimos casos, por sus peculiares características, los integrantes de tales cooperativas suelen no tener conciencia clara del significado de la entidad a la que pertenecen pues, en todo caso, su preocupación fundamental consiste en no perder o en obtener una ocupación rentada, y la cooperativa aparece como una opción forzosa para lograrlo; de allí que con frecuencia consideren a ella como su "empleador".

En ese contexto, la regulación legal de las cooperativas de trabajo surge como necesaria pero a la vez complicada, máxime cuando ellas pueden convertirse en un instrumento utilizado por los empleadores en fraude a la legislación laboral cuando éstos organizan cooperativas de trabajo con su personal a fin de eludir las obligaciones laborales y de seguridad social con fundamento en la inexistencia de vínculo de trabajo dependiente. ${ }^{35} \mathrm{De}$ allí que la OIT haya sido particularmente severa en condenar este uso espurio de las cooperativas de trabajo, señalando que las políticas nacionales deberían "velar por que no se puedan crear o utilizar cooperativas para evadir la legislación del trabajo ni ello sirva para establecer relaciones de trabajo encubiertas, y luchar contra las pseudo-cooperativas, que violan los derechos de los trabajadores, velando por que la legislación del trabajo se aplique en todas las empresas". ${ }^{36}$ Estos excesos han conducido a que en varios países las cooperativas de trabajo fueran objeto de persecución por parte de los gobiernos, sin distinguir entre las auténticas y las falsas y sin realizar una adecuada labor de control por parte de los organismos competentes.

j. Las cooperativas y los impuestos. El régimen fiscal no constituye materia propia de la legislación cooperativa pero el régimen tributario incide de manera preponderante en el desarrollo de las cooperativas y a veces se encuentra tratado en la propia ley de cooperativas.

La cuestión de fondo consiste en que se le otorgue a las cooperativas el tratamiento fiscal que corresponde a su naturaleza; es decir que no se las confunda con empresas de capital lucrativo y por esa vía se pretenda gravarlas de igual modo que a éstas. No se trata de brindarles un trato preferencial sino de considerarlas según sus propias características y de no tratar igual a empresas diferentes. Este tratamiento adecuado a su naturaleza debe estar

\footnotetext{
${ }^{34}$ Pueden mencionarse como muestra las cooperativas de trabajo promovidas por el Ministerio de Desarrollo Social argentino a través de diversos planes sociales creados desde 2003 en adelante.

${ }^{35}$ El fenómeno de la tercerización del trabajo surgió con ímpetu en la década de 1990 como forma de reducir los costos laborales y las cooperativas de trabajo fueron utilizadas con ese propósito con el consiguiente desprestigio de la figura.

${ }^{36}$ OIT, Recomendación $\mathrm{N}^{\circ} 193$, punto 8. 1) b).
}

REVESCO N 117 - MONOGRÁFICO: Las sociedades cooperativas construyen un mundo mejor - ISSN: 1885-8031 - www.ucm.es/info/revesco 
presente en las distintas clases de tributos: los que gravan los patrimonios; los que recaen sobre las rentas y los que inciden sobre las transacciones (Cracogna, 2003 y 2004).

Cuestión diferente es la relativa a las exoneraciones o desgravaciones que el Estado resuelva acordar a las cooperativas en atención a los beneficios que derivan de sus actividades, la cual corresponden de manera exclusiva a la política fiscal que en cada caso se adopte.

\section{LA LEY MARCO PARA LAS COOPERATIVAS DE AMÉRICA LATINA}

Entre las acciones posibles para alcanzar los objetivos definidos en cuanto a los marcos legales el Plan menciona brindar colaboración a los legisladores mediante estudios que faciliten su labor y expresamente alude a la Ley Marco para las Cooperativas de América Latina. Este documento fue originariamente elaborado por la Organización de las Cooperativas de América (OCA) en 1988 y actualizado veinte años más tarde por la ACI Américas con el propósito de que sirviera de orientación a los legisladores y al propio movimiento cooperativo de los países del Continente al momento de modificar o actualizar las leyes de cooperativas (Cracogna, 2009).

El documento, que se halla redactado como un texto legal y con la justificación de cada uno de sus artículos, se funda en la experiencia cooperativa continental y toma en cuenta la Declaración sobre la Identidad Cooperativa y los pronunciamientos de los organismos internacionales antes referidos. No pretende convertirse en un modelo a copiar en los distintos países sino que está concebido como una orientación basada en experiencias que resultaron exitosas y adecuadas a la genuina naturaleza cooperativa.

La Ley Marco alcanzó una significativa influencia en la legislación cooperativa continental posterior a su elaboración y estimuló asimismo los desarrollos teóricos del Derecho Cooperativo. En 2012 el Parlamento Latinoamericano le dio aprobación por lo cual cabe esperar que cobre aun mayor influencia. ${ }^{37}$

\footnotetext{
${ }^{37}$ El Parlamento Latinoamericano, formado por parlamentarios designados por sus propios parlamentos nacionales, no cumple funciones legislativas sino que formula recomendaciones dirigidas a estos últimos.

REVESCO No 117 - MONOGRÁFICO: Las sociedades cooperativas construyen un mundo mejor - ISSN: 1885-8031 - www.ucm.es/info/revesco
} 


\section{MECANISMOS DE COLABORACIÓN LEGISLATIVA}

Otros mecanismos de colaboración con los legisladores para el progreso de la legislación cooperativa consisten en el acercamiento a las distintas comisiones parlamentarias por parte de las organizaciones del movimiento cooperativo con el objetivo de brindarles información y transmitirles las inquietudes y aspiraciones de las cooperativas relacionadas con la actividad legislativa, sea en la materia específicamente cooperativa o en otras vinculadas con sus diferentes actividades. Son varios los organismos legislativos de países de la región que cuentan con comisiones especializadas en materia de cooperativas con las cuales puede llevarse a cabo una importante labor de incidencia. ${ }^{38}$

Por otra parte, en años recientes, especialmente merced a una acción que viene cumpliendo la ACI Américas, han comenzado a formarse grupos o redes nacionales de parlamentarios que acogen y promueven las iniciativas legislativas de interés para el movimiento cooperativo. Incluso existe la iniciativa de creación de una red continental de parlamentarios cooperativistas surgida a partir de las reuniones de parlamentarios realizadas por la ACI Américas durante los últimos años en coincidencia con sus conferencias regionales. ${ }^{39}$ Todas estas acciones encuadran dentro de las sugerencias contenidas en el Plan para una Década Cooperativa en materia de marcos jurídicos.

En la misma dirección se ordenan las actividades académicas de investigación y docencia universitaria en Derecho Cooperativo a fin de promover la formación de los profesionales del derecho en este campo, acerca de lo cual es muy poco lo realizado hasta el presente. También los congresos continentales de Derecho Cooperativo constituyen otro medio de contribuir al progreso de la materia. ${ }^{40}$

\footnotetext{
${ }^{38}$ El tema de la incidencia ha sido desarrollado por la ACI Américas en seminarios y cursos desarrollados en diferentes países y mediante publicaciones que pueden consultarse en www.aciamericas.coop

${ }^{39}$ Las Reuniones de Parlamentarios Cooperativistas tuvieron lugar en las conferencias regionales de la ACI Américas realizadas en México, Costa Rica, Perú, Buenos Aires y Panamá. En ellas se trataron diversos temas de interés legislativo en la región y se suscribieron documentos de conclusiones y recomendaciones.

${ }^{40}$ OCA promovió la realización de estos congresos en Mérida (Venezuela), 1969; San Juan de Puerto Rico, 1976; Rosario (Argentina), 1986 y Brasilia, 1992, cuyos documentos y declaraciones contribuyeron eficazmente al desarrollo del Derecho Cooperativo. La ACI Américas, por su parte, realizó un nuevo congreso continental en octubre de 2013 en Guarujá (Brasil) en coincidencia con su XVIII Conferencia Regional.
}

REVESCO No 117 - MONOGRÁFICO: Las sociedades cooperativas construyen un mundo mejor - ISSN: 1885-8031 - www.ucm.es/info/revesco 


\section{CONCLUSIÓN}

A esta altura resulta innegable la importancia de legislación cooperativa para posibilitar y asegurar el desarrollo de las cooperativas con adecuación a su naturaleza específica, evitando confusión con organizaciones de capital lucrativo. ${ }^{41}$ Asimismo debe garantizar la autonomía de las cooperativas, especialmente frente al Estado, y asegurarles un tratamiento no discriminatorio.

Por lo tanto, resulta comprensible el lugar de creciente interés que el tema ha ganado en el seno de la ACI y en los organismos internacionales. No extraña, pues, que el Plan para una Década Cooperativa incluya a los marcos jurídicos como un condicionante de relevancia para el logro de los objetivos que propone.

En América Latina, pese a los progresos realizados, la legislación cooperativa sigue necesitando una adecuada puesta al día, especialmente en ciertos temas como los que se han tratado en este trabajo. Para lograrlo, es necesaria una conveniente profundización de los estudios vinculados con el Derecho Cooperativo y una franca colaboración del movimiento cooperativo con los legisladores. ${ }^{42}$

\section{BIBLIOGRAFÍA}

AA.VV. (1995) Los principios cooperativos en los ordenamientos jurídicos nacionales, Boletín de la Asociación Internacional de Derecho Cooperativo, № 23/24, Bilbao.

ALIANZA COOPERATIVA INTERNACIONAL (1996) Los principios cooperativos para el Siglo XXI, Buenos Aires: Intercoop.

ACI AMÉRICAS, Ley Marco para las Cooperativas de América Latina (puede consultarse en www.aciamericas.coop )

\section{ALIANZA COOPERATIVA INTERNACIONAL, Plan para una Década Cooperativa} (puede ser consultado en www.ica.coop)

\footnotetext{
41 Sobre las tendencias actuales del Derecho Cooperativo pueden verse: Henrÿ. Hagen, "El Derecho Cooperativo. Propuestas para el Siglo XXI", en Revista de la Cooperación Internacional, Intercoop, Buenos Aires, $N^{\circ}$ 1/2010, p. 8 y ss. y Cracogna, Dante, "La legislación cooperativa en el Siglo XXI", en Cooperativismo y Desarrollo, Universidad Cooperativa de Colombia, $\mathrm{N}^{\circ} 100$, Bogotá, 2012, p. 172 y ss.

${ }^{42}$ Una fuente actual de orientación en la materia con perspectiva internacional y comparada es Cracogna, Dante - Fici, Antonio - Henrÿ, Hagen (Editors), International Handbook of Co-operative Law, Springer, Heidelberg, 2013, que contiene estudios sobre la legislación cooperativa en más de treinta países y legislación supranacional sobre la materia.
}

REVESCO No 117 - MONOGRÁFICO: Las sociedades cooperativas construyen un mundo mejor - ISSN: 1885-8031 - www.ucm.es/info/revesco 
BIRCHALL, J. y HAMMOND KETILSON, L. (2009) Resilience of the Cooperative Business Model, Geneva: ILO, 2009

BONNER, A. (1961) British Co-operation, Co-operative Union, Manchester.

CELAYA ULIBARRI, A. (1992) Capital y sociedad cooperativa, Madrid: Tecnos.

CRACOGNA, D. (1993) The relationship between the State and Cooperatives in cooperative development, informe al coloquio realizado por la OIT en Ginebra, 14 y 15 de diciembre de 1993

CRACOGNA, D. (1998) La promoción del movimiento cooperativo a través de la acción gubernamental en América Latina y el Caribe, Cuadernos de Economía Social, Instituto Argentino de Economía Social (Filial del CIRIEC), N 30, Buenos Aires.

CRACOGNA, D. (2003) Tax Exemption for Cooperatives: When and Why? The Mercosur Experience, ICA Legislative Forum, Oslo, $1^{\text {st }}$ September.

CRACOGNA, D. (2004) O acto cooperativo na América Latina. En Krueger, G. Ato Cooperativo e seu adequado tratamento tributário, Mandamentos: Belo Horizonte

CRACOGNA, D. (2004) Las cooperativas frente al régimen tributario. En Cracogna, D. (Coordinador) Las cooperativas y los impuestos en el Mercosur, Buenos Aires: Intercoop. CRACOGNA, D. (2009) Nueva versión de la Ley Marco para las Cooperativas de América Latina, Revista Jurídica de Economía Social y Cooperativa, CIRIEC-España, Valencia, $\mathrm{N}^{\mathrm{o}} 20$.

CRACOGNA, D. (2012) El capital en las cooperativas y las NIIF. en Favier Dubois, E.M. (Director) Derecho contable aplicado, Buenos Aires: Errepar.

CRACOGNA, D. (2012) La legislación cooperativa en el Siglo XXI, Cooperativismo y Desarrollo, Universidad Cooperativa de Colombia, $\mathrm{N}^{\circ} 100$.

CRACOGNA, D. (2013) Estado, cooperativas y legislación cooperativa en la hora actual. Boletín de la Asociación Internacional de Derecho Cooperativo, № 47, Bilbao.

CRACOGNA, D. - FICI, A. - HENRŸ, H. (Editors) (2013) International Handbook of Cooperative Law, Heidelberg: Springer.

FONTENLA, E.H. (2008) Cooperativas de trabajo y empresas recuperadas, Buenos Aires: Intercoop.

HENRŸ, H. (2001) Lineamientos para la legislación cooperativa, Revista de la Cooperación Internacional, Alianza Cooperativa Internacional, Intercoop, Buenos Aires, $\mathrm{N}^{\mathrm{o}} 2$.

HENRŸ, H. (2005) Guidelines for Cooperative Legislation, $2^{\text {nd }}$ edition, Geneva: ILO.

REVESCO No 117 - MONOGRÁFICO: Las sociedades cooperativas construyen un mundo mejor - ISSN: 1885-8031 - www.ucm.es/info/revesco 
HENRŸ, H. (2010) El Derecho Cooperativo. Propuestas para el Siglo XXI, Revista de la Cooperación Internacional, Intercoop, Buenos Aires, $N^{\circ}$ 1/2010

MONTOLÍO, J.M. (2011) Legislación cooperativa mundial. Tendencias y perspectivas en América Latina, Boletín de la Asociación Internacional de Derecho Cooperativo, Bilbao, $\mathrm{N}^{\circ} 45$.

MÜNKNER, H-H. (1985) Co-operative Principles and Co-operative Law, Friedrich-EbertStiftung, Bonn.

MÜNKNER, H-H. (2005) The 'Classical British Indian Pattern of Co-operation' from statesponsorship to state-control. En Münkner, H-H. (Editor), 100 Years Co-operative Credit Societies Act India 1904. A worldwide applied model of co-operative legislation, Marburg Consult for Self-help Promotion: Marburg.

OIT, Recomendación No 193 sobre Promoción de las Cooperativas

ONU, Resolución 56/ 114 de la Asamblea General de 2001

ONU, Resolución 64/136sesión plenaria del 18.12.09

PASTOR SEMPERE, C. (2002) Los recursos propios en las sociedades cooperativas, Madrid: Editoriales de Derecho Reunidas.

TEVEZ, A.N. (2010) Empresas recuperadas y cooperativas de trabajo, Buenos Aires: Astrea.

VALKO, L. (1952) The First Co-operative Law, Review of International Cooperation, International Cooperative Alliance, London, reproducido en Valko, Laszlo, Essays on Modern Co-operation, Washington State University Press, 1964

VALKO, L. (1954) International Handbook of Cooperative Legislation, State College of Washington.

WATKINS, W.P (1990) Co-operative Principles Today and Tomorrow, Manchester: Holyoake Books.

REVESCO No 117 - MONOGRÁFICO: Las sociedades cooperativas construyen un mundo mejor - ISSN: 1885-8031 - www.ucm.es/info/revesco 